\title{
Diagnostic Value of Knee Arthrometry in the Prediction of Anterior Cruciate Ligament Strain During Landing
}

\author{
Ata M. Kiapour, $\mathrm{PhD}^{\star}, \dagger$, Samuel C. Wordeman, BS $\ddagger, \S$, Mark V. Paterno, PT, PhD, SCS, \\ ATC $\|$, , Carmen E. Quatman, MD, PhD ${ }^{\ddagger}, \#$, Jason W. Levine, $\mathrm{MD}^{\dagger}$, Vijay K. Goel, $\mathrm{PhD}^{\dagger}$, \\ Constantine K. Demetropoulos, $\mathrm{PhD}^{\star *}$, and Timothy E. Hewett, $\mathrm{PhD}^{\ddagger, \S, q, \#, \uparrow \dagger, \ddagger \ddagger}$
}

\begin{abstract}
*Sports Medicine Research Laboratory, Department of Orthopaedic Surgery, Boston Children's Hospital, Harvard Medical School, Boston, Massachusetts. 'Engineering Center for Orthopaedic Research Excellence (ECORE), Departments of Orthopaedics and Bioengineering, The University of Toledo, Toledo, Ohio. ¥Sports Health and Performance Institute, The Ohio State University, Columbus, Ohio. §Department of Biomedical Engineering, The Ohio State University, Columbus, Ohio. "Division of Occupational Therapy and Physical Therapy, Cincinnati Children's Hospital Medical Center, Cincinnati, Ohio. "Sports Medicine Biodynamics Center, Cincinnati Children's Hospital Medical Center, Cincinnati, Ohio. \#Department of Orthopaedic Surgery, The Ohio State University, Columbus, Ohio. ${ }^{* *}$ Biomechanics \& Injury Mitigation Systems, Research \& Exploratory Development Department, The Johns Hopkins University Applied Physics Laboratory, Laurel, Maryland. ${ }^{+t}$ Departments of Physiology and Cell Biology and Family Medicine and the School of Health and Rehabilitation Sciences, The Ohio State University, Columbus, Ohio.
\end{abstract}

\begin{abstract}
Background-Previous studies have indicated that higher knee joint laxity may be indicative of an increased risk of anterior cruciate ligament (ACL) injuries. Despite the frequent clinical use of knee arthrometry in the evaluation of knee laxity, little data exist to correlate instrumented laxity measures and ACL strain during dynamic high-risk activities.
\end{abstract}

Purpose/Hypotheses-The purpose of this study was to evaluate the relationships between ACL strain and anterior knee laxity measurements using arthrometry during both a drawer test and simulated bipedal landing (as an identified high-risk injurious task). We hypothesized that a high correlation exists between dynamic ACL strain and passive arthrometry displacement. The secondary hypothesis was that anterior knee laxity quantified by knee arthrometry is a valid predictor of injury risk such that specimens with greater anterior knee laxity would demonstrate increased levels of peak ACL strain during landing.

Study Design-Controlled laboratory study.

Methods-Twenty cadaveric lower limbs (mean age, $46 \pm 6$ years; 10 female and 10 male) were tested using a CompuKT knee arthrometer to measure knee joint laxity. Each specimen was tested under 4 continuous cycles of anterior-posterior shear force $( \pm 134 \mathrm{~N})$ applied to the tibial tubercle. To quantify ACL strain, a differential variable reluctance transducer (DVRT) was arthroscopically

\footnotetext{
(C) 2013 The Author(s)

¥¥Address correspondence to Timothy E. Hewett, PhD, Sports Health and Performance Institute, The Ohio State University, 2050 Kenny Road, Suite 3100, Columbus, OH 43221 (Timothy.Hewett@ osumc.edu).

Investigation performed at the Engineering Center for Orthopaedic Research Excellence (ECORE), The University of Toledo, Toledo, Ohio

Presented as a poster at the 39th annual meeting of the AOSSM, Chicago, Illinois, July 2013.

For reprints and permission queries, please visit SAGE's Web site at http://www.sagepub.com/journalsPermissions.nav
} 
placed on the ACL (anteromedial bundle), and specimens were retested. Subsequently, bipedal landing from $30 \mathrm{~cm}$ was simulated in a subset of 14 specimens (mean age, $45 \pm 6$ years; 6 female and 8 male) using a novel custom-designed drop stand. Changes in joint laxity and ACL strain under applied anterior shear force were statistically analyzed using paired sample $t$ tests and analysis of variance. Multiple linear regression analyses were conducted to determine the relationship between anterior shear force, anterior tibial translation, and ACL strain.

Results-During simulated drawer tests, $134 \mathrm{~N}$ of applied anterior shear load produced a mean peak anterior tibial translation of $3.1 \pm 1.1 \mathrm{~mm}$ and a mean peak ACL strain of $4.9 \% \pm 4.3 \%$.

Anterior shear load was a significant determinant of anterior tibial translation $(P<.0005)$ and peak ACL strain $(P=.04)$. A significant correlation $(r=0.52, P<.0005)$ was observed between anterior tibial translation and ACL strain. Cadaveric simulations of landing produced a mean axial impact load of $4070 \pm 732 \mathrm{~N}$. Simulated landing significantly increased the mean peak anterior tibial translation to $10.4 \pm 3.5 \mathrm{~mm}$ and the mean peak ACL strain to $6.8 \% \pm 2.8 \%(P<.0005)$ compared with the prelanding condition. Significant correlations were observed between peak ACL strain during simulated landing and anterior tibial translation quantified by knee arthrometry.

Conclusion-Our first hypothesis is supported by a significant correlation between arthrometry displacement collected during laxity tests and concurrent ACL strain calculated from DVRT measurements. Experimental findings also support our second hypothesis that instrumented measures of anterior knee laxity predict peak ACL strain during landing, while specimens with greater knee laxity demonstrated higher levels of peak ACL strain during landing.

Clinical Relevance-The current findings highlight the importance of instrumented anterior knee laxity assessments as a potential indicator of the risk of ACL injuries in addition to its clinical utility in the evaluation of ACL integrity.

\section{Keywords}

ACL; knee; laxity; arthrometry; injury

The anterior cruciate ligament (ACL) is the primary passive restraint to anterior translation of the tibia with respect to the femur. ${ }^{1,4,11,19,20} \mathrm{~A}$ cadaveric study by Butler et $\mathrm{al}^{4}$ demonstrated that the ACL provides $85 \%$ to $87 \%$ of the passive anterior tibial restraint at knee flexion angles of approximately $20^{\circ}$ to $30^{\circ}$. Dynamic knee stability is affected by both passive (ligamentous) and active (neuromuscular) joint restraints. ${ }^{29,30}$ The increased physiological laxity of the knee joint may contribute to deficits in dynamic joint stability and, as a result, an increased risk of ACL injuries during high-risk maneuvers. ${ }^{23,28,31-33}$

Instrumented measurements of anterior-posterior (A-P) knee laxity, or arthrometry, are most often assessed during anterior drawer and Lachman tests. Knee arthrometers offer objective, quantitative, and accurate information that cannot be obtained from a Lachman examination. These methods are well established as diagnostic clinical tools to assess ACL integrity as well as in research to provide objective measures of passive joint stability. ${ }^{23,34}$ Previous prospective clinical studies have reported that higher knee joint laxity may indicate an increased risk of ACL injuries. ${ }^{23,28,31-33}$ However, no studies have determined the correlation between knee joint laxity and ACL strain (as a measure of the risk of ACL injuries) during high-risk maneuvers. Torry and colleagues ${ }^{31}$ have reported a strong correlation between passive anterior knee laxity, measured by knee arthrometry, and peak anterior tibial translation, measured in vivo during dynamic drop landing, in 16 healthy adults. They concluded that anterior laxity can be considered as an established parameter in evaluating the risk of noncontact ACL injuries. In a prospective 2-year study of 44 high school and college athletes, Woodford-Rogers and colleagues ${ }^{33}$ reported substantially greater knee laxity within the ACL-injured group (obtained from the contralateral uninjured 
knee) compared with the athletes with intact ACLs. They further demonstrated that knee laxity is among the best predictors of athletes' group classification (ACL-injured or ACLintact). Finally, they reported that greater knee joint laxity may result in an elevated risk of ACL injuries. Uhorchak et $\mathrm{al}^{32}$ reported that female athletes with a higher passive joint restraint, defined as generalized joint laxity greater than 1 standard deviation above the mean, demonstrated an increased risk of ACL injuries. Similarly, Ramesh et $\mathrm{al}^{28}$ observed increased rates of ACL injuries in patients with increased overall joint laxity, specifically those with increased knee joint laxity. Further, a prospective study of 1558 young female athletes by Myer et al ${ }^{23}$ reported a significant correlation between side-to-side differences in passive A-P knee joint laxity and the potential risk of ACL injuries. They have suggested that increased passive A-P laxity of the knee joint may be associated with an increased risk of ACL injuries.

Despite the frequent clinical use of knee arthrometry, little data exist to correlate arthrometric measurements and ACL strain. The purpose of this study was to evaluate the relationships between anterior knee laxity measurements and ACL strain under both a passive drawer test and simulated bipedal landing (as an identified high-risk injurious task) using a cadaveric model. We hypothesized that a high correlation exists between arthrometry displacement and ACL strain during the laxity test. The secondary hypothesis was that specimens with higher anterior knee laxity would demonstrate higher levels of peak ACL strain during landing.

\section{MATERIALS AND METHODS}

\section{Knee Arthrometry (Passive Drawer Test)}

Twenty unembalmed, fresh-frozen cadaveric lower limbs (mean age, $46 \pm 6$ years; 10 female and 10 male) were acquired. Specimens were inspected visually and by computed tomography and magnetic resonance imaging by a board-certified orthopaedic surgeon for signs of soft or hard tissue injuries including indications of surgery, deformities, and soft tissue disruption. Also, A-P knee laxity of each specimen was evaluated using a CompuKT knee arthrometer (MEDmetric Corp, San Diego, California). Specimens were positioned to simulate a supine position with the knees stabilized at $25^{\circ}$ of flexion over a standard adjustable thigh support (Figure 1). The arthrometer was secured to the shank, such that the patellar sensor pad rested on the patella with the specimens aligned with the knee joint line's reference mark on the arthrometer. The knee was placed in neutral alignment using the support bar included with the arthrometry kit. The ankle and foot were stabilized to restrict tibial rotation throughout testing per the manufacturer's recommendation..$^{5,7}$ A $134-\mathrm{N}$ posterior force was applied to establish the limit of posterior tibial displacement before application of the anterior force. Each specimen was tested under 4 continuous cycles of A-P shear force $( \pm 134 \mathrm{~N})$ applied to the tibial tubercle via the arthrometer's force-sensing handle. To quantify ACL strain during simulated drawer tests, a differential variable reluctance transducer (DVRT) (MicroStrain Inc, Williston, Vermont) was arthroscopically placed on the distal third of the anteromedial (AM) bundle through 2 parapatellar incisions (Figure 2). Subsequent to DVRT insertion, the drawer tests were repeated. To calculate absolute strain values, the ACL's reference length was calculated based on the method described by Howe et $\mathrm{al}^{14}$ and Fleming et $\mathrm{al}^{10}$ to identify the DVRT's length corresponding to the ligament-slack and -taut conditions. It was assumed that the mean strain across the ACL's AM bundle was equal to the change in length of the measured segment divided by the reference length (Kiapour AM, Quatman CE, Ditto RC, et al, "Influence of Axial Rotation Moments on ACL Strain: A Cadaveric Study of Single- and Multi-Axis Loading of the Knee." Paper presented at the proceedings of 37th ASB annual meeting, 2012). ${ }^{16}$ All instrumented knee joint drawer tests were performed by a single licensed physical therapist 
(intrarater reliability [intraclass correlation coefficient $]=0.92) .{ }^{27}$ Analog data (both arthrometer and DVRT) were collected at $100 \mathrm{~Hz}$.

\section{Dynamic Testing (Cadaveric Simulation of Bipedal Landing)}

Subsequent to arthrometry, a subset of 14 specimens (mean age, $45 \pm 6$ years; 6 female and 8 male) were tested under dynamic conditions. It is noteworthy to mention that 6 of 20 specimens used in the first part of the study were randomly selected for multiple trials to better design and optimize the dynamic test setup and loading protocol. These 6 trial specimens were eliminated from the second set of the experiments (dynamic testing) because of tissue damage and ACL failure during trials and the tune-up process. Specimens were stored at $-20^{\circ} \mathrm{C}$. Before testing, specimens were slowly thawed to room temperature. Specimens were sectioned at the midfemoral diaphysis ( $30 \mathrm{~cm}$ above the joint line), and all soft tissues up to $15 \mathrm{~cm}$ proximal to the joint line were dissected. The remaining bony structure of the proximal femur of each specimen was potted for rigid attachment to the testing frame. The quadriceps (rectus femoris) and hamstring (semitendinosus, biceps femoris, and semimembranosus) tendons were then isolated and clamped inside metal tendon grips to allow for the application of simulated muscle loads. The musculature and skin directly around the knee joint were maintained intact. The foot and ankle were also maintained intact to provide a realistic load-transfer interface. Saline solution was used throughout specimen preparation and testing to maintain tissue hydration.

A novel custom-designed drop stand was used to simulate dynamic landing. ${ }^{17,26}$ Specimens were mounted in such a way to simulate lower extremity postures during ground strike, while landing from a jump with knees flexed at $25^{\circ}$ (Figure 3 ). Each specimen was rigidly fixed at the proximal femur, and the tibia was orientated vertically with the foot above. Transknee muscle forces were simulated utilizing multiple cable-pulley systems along with static weights to apply constant forces to the quadriceps (1200 N) and hamstring (800 N). Adjustable pulley systems were used to maintain the physiological line of action of each muscle (Figure 3). An athletic shoe was placed on the foot to provide realistic load transfer during initial contact. Subsequent to the application of muscle loads, the floor platform was set upon the shoe to simulate a foot-planted position. Bipedal landing was simulated by the release of half the body weight $(350 \mathrm{~N})$ from a height of $30 \mathrm{~cm}$ utilizing a hemispherical impactor with an integrated weight stack. The drop weight exerted an impulsive axial compressive force that simulated the ground-reaction force during landing from a jump. Moreover, ACL strain was calculated based on the measurements of the DVRT sensor on the ACL's AM bundle. The tibiofemoral joint kinematics were captured using an Optotrak 3020 3-dimensional motion capture system (Northern Digital, Waterloo, Ontario, Canada) along with 2 arrays of 3 infrared light-emitting diode markers rigidly attached to the femur and tibia. The obtained coordinates from each marker were then transferred to a joint local coordinate system to obtain anatomic joint kinematics. ${ }^{35}$ The initial position of the tibia with respect to the femur in an unloaded position was considered as the reference frame in the calculation of joint kinematics. The knee joint rotations were defined as Euler angles of the tibia's reference frame relative to the femur's reference frame. Two 6-axis load cells (RA Denton Corp, Rochester Hills, Michigan) incorporated within the floor pad and femoral fixture were used to capture all forces and moments delivered to the knee joint and reaction forces and moments during simulated landings. Data collection from all data acquisition units was synchronized utilizing a simultaneous triggering system. Analog data (load cell and DVRT) were collected at $4 \mathrm{kHz}$, while motion data were collected at $400 \mathrm{~Hz}$.

\section{Statistical Analysis}

Paired sample $t$ tests were used to investigate changes in both anterior tibial translation and ACL strain under simulated passive drawer tests (before and after DVRT insertion) and 
bipedal landing (before landing and peak). An analysis of variance (ANOVA) with a post hoc Bonferroni correction for multiple comparisons was used to study the relationships between applied shear force, joint laxity, and ACL strain. Multiple linear regression analyses were conducted to determine the correlation between anterior drawer load, anterior tibial translation, and ACL strain. Correlations were classified as poor $(<0.4)$, good $(0.4-0.74)$, and strong ( $\searrow$.75) based on the determined Pearson correlation coefficient $(r) .{ }^{25}$ Differences were considered to be statistically significant for $P<.05$.

\section{RESULTS}

A summary of the mean anterior tibial translation for both before and after DVRT insertion along with the mean calculated ACL strain during simulated drawer tests is presented in Table 1. No significant difference in anterior tibial translation was observed before and after DVRT insertion on the ACL's AM bundle under $67 \mathrm{~N}(P=.92), 89 \mathrm{~N}(P=.98)$, and $134 \mathrm{~N}$ $(P=.41)$ of anterior drawer force. During knee arthrometry (simulated passive drawer tests), an increased anterior shear force resulted in significantly greater anterior tibial translation (anterior knee laxity) and mean ACL strain (Figure 4). Further, linear regression analysis with the assumption that 0 -mm anterior tibial translation corresponds with $0 \%$ ACL strain $(\mathrm{y}$-intercept $=0)$ demonstrated a good statistically significant correlation $(r=0.52, P<$. 0005) between arthrometer-measured anterior knee laxity and resultant ACL strain (Figure $5)$.

A summary of peak axial impact force, anterior tibial translation, and ACL strain during simulated landings is presented in Table 2. Before impact, muscle forces in the quadriceps and hamstring muscle groups produced a mean anterior tibial translation of $3.5 \pm 3.1 \mathrm{~mm}$ and ACL strain of $2.0 \% \pm 2.2 \%$. Simulated landing resulted in a mean axial impact load of $4070 \pm 732 \mathrm{~N}$ over a period of 70 milliseconds. Loads generated by axial impact increased the mean anterior tibial translation by $6.9 \pm 2.3 \mathrm{~mm}$ and increased the mean ACL strain by $4.9 \% \pm 2.6 \%$. Simulated landing significantly increased peak anterior tibial translation to $10.4 \pm 3.5 \mathrm{~mm}(P<.0005)$ and peak ACL strain to $6.8 \% \pm 2.8 \%(P<.0005)$ compared with the prelanding condition. No tissue failure was observed across anatomic structures of the knee after testing.

Significant correlations were observed between resultant peak ACL strains during simulated landing and anterior tibial translation under anterior shear forces (during simulated drawer tests) of $67 \mathrm{~N}(r=0.59, P=.02), 89 \mathrm{~N}(r=0.57, P=.03)$, and $134 \mathrm{~N}(r=0.62, P=.01)$. It was further shown that the specimens with higher magnitudes of anterior tibial translation during knee arthrometry testing demonstrate greater levels of peak ACL strain during simulated landings (Figure 6). To better understand the correlation between the risk of ACL injuries (measure by peak ACL strain) and quantified anterior knee laxity, graphs presented in Figure 6 were divided into 4 quadrants by 2 lines representing the mean values of anterior tibial translation (vertical line) and peak ACL strain (horizontal line) for the tested specimens. A substantial number of the specimens $(67 \%, 72 \%$, and $83 \%$ under $67-\mathrm{N}, 89-\mathrm{N}$, and 134-N shear loads, respectively) with anterior knee laxity greater than the mean were located at the first quadrant (top right, high-risk zone), demonstrating greater peak ACL strain than the mean during landing. However, the majority of the specimens $(75 \%, 86 \%$, and $88 \%$ under $67-\mathrm{N}, 89-\mathrm{N}$, and $134-\mathrm{N}$ shear loads, respectively) with anterior knee laxity less than the mean were located in the third quadrant (bottom left, low-risk zone), demonstrating lower peak ACL strain than the mean during simulated landing. 


\section{DISCUSSION}

Injuries of the ACL are exceedingly common and often devastating to adolescents and young adults. ${ }^{6,12}$ The associated costs and resulting long-term disability have generated significant interest in the investigation of non-contact ACL injury mechanisms. Landing after a jump has been identified as a high-risk task that may, under certain conditions, lead to noncontact ACL injuries. ${ }^{3,15,24}$ Instrumented A-P knee laxity tests are well-established and reliable techniques that have been used extensively by clinicians to quantify knee joint laxity and to evaluate ACL integrity. Previous prospective clinical investigations have demonstrated that patients with higher knee laxity are at an increased risk of ACL injuries. $^{23,28,31-33}$ Despite substantial research on the effects of knee joint laxity and the risk of ACL injuries, the biomechanical relationship between anterior knee laxity and ACL strain (as a measure of the risk of ACL injuries) during high-risk activities has yet to be established. This study aimed to investigate the correlation between anterior knee laxity and ACL strain during a high-risk dynamic landing task using a cadaveric model.

Knee arthrometry was used to quantify the A-P knee laxity of 20 relatively young, intact cadaveric lower limbs with and without DVRTs. The insertion of DVRTs across the AM bundle of the ACL resulted in no significant difference in instrumented laxity measures. Under simulated drawer tests, applied anterior shear forces were shown to be a significant factor in anterior tibial translation (anterior knee laxity) and resultant ACL strain levels. It was demonstrated that greater anterior shear forces can lead to increases in anterior tibial translation and ACL strain. This is in agreement with previous findings that demonstrate that the ACL provides up to $87 \%$ of the anterior tibial restraining force. ${ }^{1,4,11,19,20} \mathrm{~A}$ significant linear correlation was established between the magnitudes of anterior tibial translation and ACL strain at $25^{\circ}$ of knee flexion. This direct correlation indicates that knee arthrometry is a good indicator of ACL strain and, therefore, has intrinsic value in the evaluation of ACL loading characteristics and the diagnosis of a functionally compromised ACL. In an in vivo study of 5 human patients, Fleming et $\mathrm{al}^{9}$ established a similar correlation $(r=0.59)$ between anterior tibial translation and ACL strain (measured by a Hall effect transducer placed on the ACL's AM bundle) during passive laxity testing.

Subsequent to the instrumented laxity evaluation, bipedal landings after a jump from a 30$\mathrm{cm}$ height were simulated on a subset of 14 specimens. Results demonstrated the ability of axial impact loads (that simulate the vertical component of the ground-reaction force) to generate anterior translation of the tibia with respect to the femur. The impact-induced anterior translation of the tibia relative to the femur can be biomechanically described as the tibiofemoral joint reaction to generated shear forces due to the specific anatomy of the tibial plateau under shallow knee flexion angles, as demonstrated by other investigators. ${ }^{8,21,22}$ As expected, the impact-induced anterior tibial translation resulted in elevated ACL strain levels after peak axial impact.

Significant direct linear relationships between the assessed anterior knee laxity after arthrometry and peak ACL strain during simulated landings were observed. Specimens with greater anterior knee laxity demonstrated an increased risk of ACL injuries, as the majority of these specimens were located within the high-risk zone. In contrast, specimens with lower anterior knee laxity had a lower risk of ACL injuries, as the majority of these were located within the low-risk zone (Figure 6). Although no studies have been able to directly quantify ACL strain during landing from a jump in vivo, the results from this study indicate that patients with increased anterior knee laxity may experience higher ACL strains during landing, which may place them at an increased risk of ACL injuries. 
This study is the first to establish a significant correlation between anterior knee joint laxity measured by arthrometry and peak ACL strain during simulated dynamic landing conditions. The instrumented evaluation of A-P knee laxity has been identified as one of the most common, accurate, and valid approaches to evaluate ACL integrity. The CompuKT arthrometer has been used extensively to quantify knee joint laxity and demonstrates goodto-excellent intrarater reliability. ${ }^{34}$ Considering ACL strain as an established quantifiable measure for the risk of ACL injuries, the current findings highlight the importance of instrumented knee A-P laxity assessment as a potential indicator of the risk of ACL injuries in addition to its clinical utility in the evaluation of ACL integrity.

\section{Study Limitations}

Despite the strong findings reported, there are inherent limitations, as with any experimental study. As noted by Gross et al, ${ }^{13}$ a number of factors, including arthrometer placement, flexion angle, and speed and angle of force application may influence variables quantified during instrumented laxity testing. We have attempted to minimize these problems and associated measurement errors by having a single, highly reliable licensed physical therapist, with over 15 years of experience with the CompuKT knee arthrometer, collect all arthrometry measurements. Further, the change in A-P knee translation due to muscle cocontraction during knee arthrometry testing in vivo has been identified as one of the main limitations of this technique. However, the use of CompuKT arthrometry in the current cadaveric work is well justified because of the lack of any active neuromuscular controls. Moreover, the use of the CompuKT knee arthrometer has provided the operator with the advantage of live visual feedback regarding the curve shape and test consistency compared with traditional knee arthrometers (ie, KT1000 arthrometer). This would increase the reliability of the results by allowing the operator to adjust the placement/pull angle based on the observed live feedback. Finally, the lack of significant differences between data before and after DVRT insertion demonstrates consistent measurements using this approach in addition to demonstrating that the minimally invasive placement of DVRTs did not affect knee laxity measures. Another limitation was the potential differences in tissue properties associated with cadaveric specimens compared with in vivo tissue properties of young athletes, which can affect the accuracy of absolute reported values. We have tried to minimize this artifact by testing relatively young specimens (mean age, $46 \pm 6$ years). Further, ACL strain was represented only by local strain measurements across the AM bundle. The attachment of a second DVRT to the posterolateral bundle of the ACL would have been associated with compromise of the posterior joint capsule and potential measurement artifacts. ${ }^{2}$ The choice to place a single DVRT on the ACL's AM bundle was based on previous work that found the AM bundle's strain to be a good representation of overall ACL strain. ${ }^{18}$ Finally, the utilized cadaveric model of landing was associated with a number of assumptions and simplifications including, but not limited to, static muscle loading, orientation of the ground-reaction force relative to the tibia, exclusion of transknee muscles other than the quadriceps and hamstring, and limited range of knee flexion. Care was taken to understand these limitations during the interpretation of our findings.

\section{CONCLUSION}

The current experimental findings support our first hypothesis, demonstrating a significant direct correlation between arthrometry displacement during laxity tests and concurrent ACL strain calculated from DVRT measurements. Results further support our second hypothesis that instrumented measures of anterior knee laxity predict peak ACL strain during simulated landings. It also indicates that the specimens with higher anterior knee laxity exhibit increased peak ACL strain during simulated landing. 


\section{Acknowledgments}

One or more of the authors has declared the following potential conflict of interest or source of funding: The authors acknowledge funding support from the National Institutes of Health/National Institute of Arthritis and Musculoskeletal and Skin Diseases grants R01-AR049735 and R01-AR056259.

The authors thank Nathaniel Bates for his assistance.

\section{References}

1. Arms S, Boyle J, Johnson R, Pope M. Strain measurement in the medial collateral ligament of the human knee: an autopsy study. J Biomech. 1983; 16(7):491-496. [PubMed: 6619166]

2. Bach JM, Hull ML. Strain inhomogeneity in the anterior cruciate ligament under application of external and muscular loads. J Biomech Eng. 1998; 120(4):497-503. [PubMed: 10412421]

3. Boden BP, Dean GS, Feagin JA, Garrett WE. Mechanisms of anterior cruciate ligament injury. Orthopedics. 2000; 23(6):573-578. [PubMed: 10875418]

4. Butler DL, Noyes FR, Grood ES. Ligamentous restraints to anterior-posterior drawer in the human knee: a biomechanical study. J Bone Joint Surg Am. 1980; 62(2):259-270. [PubMed: 7358757]

5. Daniel DM, Malcom LL, Losse G, Stone ML, Sachs R, Burks R. Instrumented measurement of anterior laxity of the knee. J Bone Joint Surg Am. 1985; 67(5):720-726. [PubMed: 3997924]

6. Daniel DM, Stone ML, Dobson BE, Fithian DC, Rossman DJ, Kaufman KR. Fate of the ACLinjured patient: a prospective outcome study. Am J Sports Med. 1994; 22(5):632-644. [PubMed: 7810787]

7. Daniel DM, Stone ML, Sachs R, Malcom L. Instrumented measurement of anterior knee laxity in patients with acute anterior cruciate ligament disruption. Am J Sports Med. 1985; 13(6):401-407. [PubMed: 4073348]

8. Dejour H, Bonnin M. Tibial translation after anterior cruciate ligament rupture: two radiological tests compared. J Bone Joint Surg Br. 1994; 76(5):745-749. [PubMed: 8083263]

9. Fleming BC, Beynnon BD, Nichols CE, Johnson RJ, Pope MH. An in vivo comparison of anterior tibial translation and strain in the anteromedial band of the anterior cruciate ligament. J Biomech. 1993; 26(1):51-58. [PubMed: 8423168]

10. Fleming BC, Beynnon BD, Tohyama H, et al. Determination of a zero strain reference for the anteromedial band of the anterior cruciate ligament. J Orthop Res. 1994; 12(6):789-795. [PubMed: 7983554]

11. Fukubayashi T, Torzilli PA, Sherman MF, Warren RF. An in vitro biomechanical evaluation of anterior-posterior motion of the knee: tibial displacement, rotation, and torque. J Bone Joint Surg Am. 1982; 64(2):258-264. [PubMed: 7056781]

12. Griffin LY, Agel J, Albohm MJ, et al. Noncontact anterior cruciate ligament injuries: risk factors and prevention strategies. J Am Acad Orthop Surg. 2000; 8(3):141-150. [PubMed: 10874221]

13. Gross SM, Carcia CR, Gansneder BM, Shultz SJ. Rate of force application during knee arthrometer testing affects stiffness but not displacement measurements. J Orthop Sports Phys Ther. 2004; 34(3):132-139. [PubMed: 15089026]

14. Howe JG, Wertheimer C, Johnson RJ, Nichols CE, Pope MH, Beynnon B. Arthroscopic strain gauge measurement of the normal anterior cruciate ligament. Arthroscopy. 1990; 6(3):198-204. [PubMed: 2206182]

15. Kiapour, AM. dissertation. Toledo, Ohio: Department of Bioengineering, The University of Toledo; 2013. Non-Contact ACL Injuries During Landing: Risk Factors and Mechanisms.

16. Kiapour, AM.; Quatman, CE.; Ditto, RC., et al. Proceedings of 2012 ASME Summer Bioengineering Conference. Vol. 44809. Fajardo, Puerto Rico: American Society of Mechanical Engineers (ASME); 2012. Global quasi-static mechanical characterization of the human knee under single- and multi-axis unconstrained loading conditions; p. 1119-1120.

17. Levine JW, Kiapour AM, Quatman CE, et al. Clinically relevant injury patterns after an anterior cruciate ligament injury provide insight into injury mechanisms. Am J Sports Med. 2013; 41(2): 385-395. [PubMed: 23144366] 
18. Markolf KL, Gorek JF, Kabo JM, Shapiro MS. Direct measurement of resultant forces in the anterior cruciate ligament: an in vitro study performed with a new experimental technique. J Bone Joint Surg Am. 1990; 72(4):557-567. [PubMed: 2324143]

19. Markolf KL, Graff-Radford A, Amstutz HC. In vivo knee stability: a quantitative assessment using an instrumented clinical testing apparatus. J Bone Joint Surg Am. 1978; 60(5):664-674. [PubMed: 681387]

20. Markolf KL, Mensch JS, Amstutz HC. Stiffness and laxity of the knee: the contributions of the supporting structures. A quantitative in vitro study. J Bone Joint Surg Am. 1976; 58(5):583-594. [PubMed: 946969]

21. McLean SG, Oh YK, Palmer ML, et al. The relationship between anterior tibial acceleration, tibial slope, and ACL strain during a simulated jump landing task. J Bone Joint Surg Am. 2011; 93(14): 1310-1317. [PubMed: 21792497]

22. Meyer EG, Haut RC. Excessive compression of the human tibio-femoral joint causes ACL rupture. J Biomech. 2005; 38(11):2311-2316. [PubMed: 16154419]

23. Myer GD, Ford KR, Paterno MV, Nick TG, Hewett TE. The effects of generalized joint laxity on risk of anterior cruciate ligament injury in young female athletes. Am J Sports Med. 2008; 36(6): 1073-1080. [PubMed: 18326833]

24. Olsen OE, Myklebust G, Engebretsen L, Bahr R. Injury mechanisms for anterior cruciate ligament injuries in team handball: a systematic video analysis. Am J Sports Med. 2004; 32(4):1002-1012. [PubMed: 15150050]

25. Portney, LG.; Watkins, MP. Foundations of Clinical Research: Applications to Practice. 2. Upper Saddle River, New Jersey: Prentice Hall; 1999.

26. Quatman CE, Kiapour AM, Demetropoulos CK, et al. Preferential loading of the ACL compared with the MCL during landing: a novel in sim approach yields the multiplanar mechanism of dynamic valgus during ACL injuries. Am J Sports Med. published online October 11, 2013. $10.1177 / 0363546513506558$

27. Quatman CE, Paterno MV, Wordeman SC, Kaeding CC. Longitudinal anterior knee laxity related to substantial tibial tunnel enlargement after anterior cruciate ligament revision. Arthroscopy. 2011; 27(8):1160-1163. [PubMed: 21802626]

28. Ramesh R, Von Arx O, Azzopardi T, Schranz PJ. The risk of anterior cruciate ligament rupture with generalised joint laxity. J Bone Joint Surg Br. 2005; 87(6):800-803. [PubMed: 15911662]

29. Rozzi SL, Lephart SM, Gear WS, Fu FH. Knee joint laxity and neuromuscular characteristics of male and female soccer and basketball players. Am J Sports Med. 1999; 27(3):312-319. [PubMed: 10352766]

30. Shultz SJ, Carcia CR, Perrin DH. Knee joint laxity affects muscle activation patterns in the healthy knee. J Electromyogr Kinesiol. 2004; 14(4):475-483. [PubMed: 15165597]

31. Torry MR, Myers C, Pennington WW, et al. Relationship of anterior knee laxity to knee translations during drop landings: a bi-plane fluoroscopy study. Knee Surg Sports Traumatol Arthrosc. 2011; 19(4):653-662. [PubMed: 21153545]

32. Uhorchak JM, Scoville CR, Williams GN, Arciero RA, St Pierre P, Taylor DC. Risk factors associated with noncontact injury of the anterior cruciate ligament: a prospective four-year evaluation of 859 West Point cadets. Am J Sports Med. 2003; 31(6):831-842. [PubMed: 14623646]

33. Woodford-Rogers B, Cyphert L, Denegar CR. Risk factors for anterior cruciate ligament injury in high school and college athletes. J Athl Train. 1994; 29(4):343-346. [PubMed: 16558298]

34. Wordeman SC, Paterno MV, Quatman CE, Bates NA, Hewett TE. Arthrometric curve-shape variables to assess anterior cruciate ligament deficiency. Clin Biomech (Bristol, Avon). 2012; 27(8):830-836.

35. Yu B, Lin CF, Garrett WE. Lower extremity biomechanics during the landing of a stop-jump task. Clin Biomech (Bristol, Avon). 2006; 21(3):297-305. 


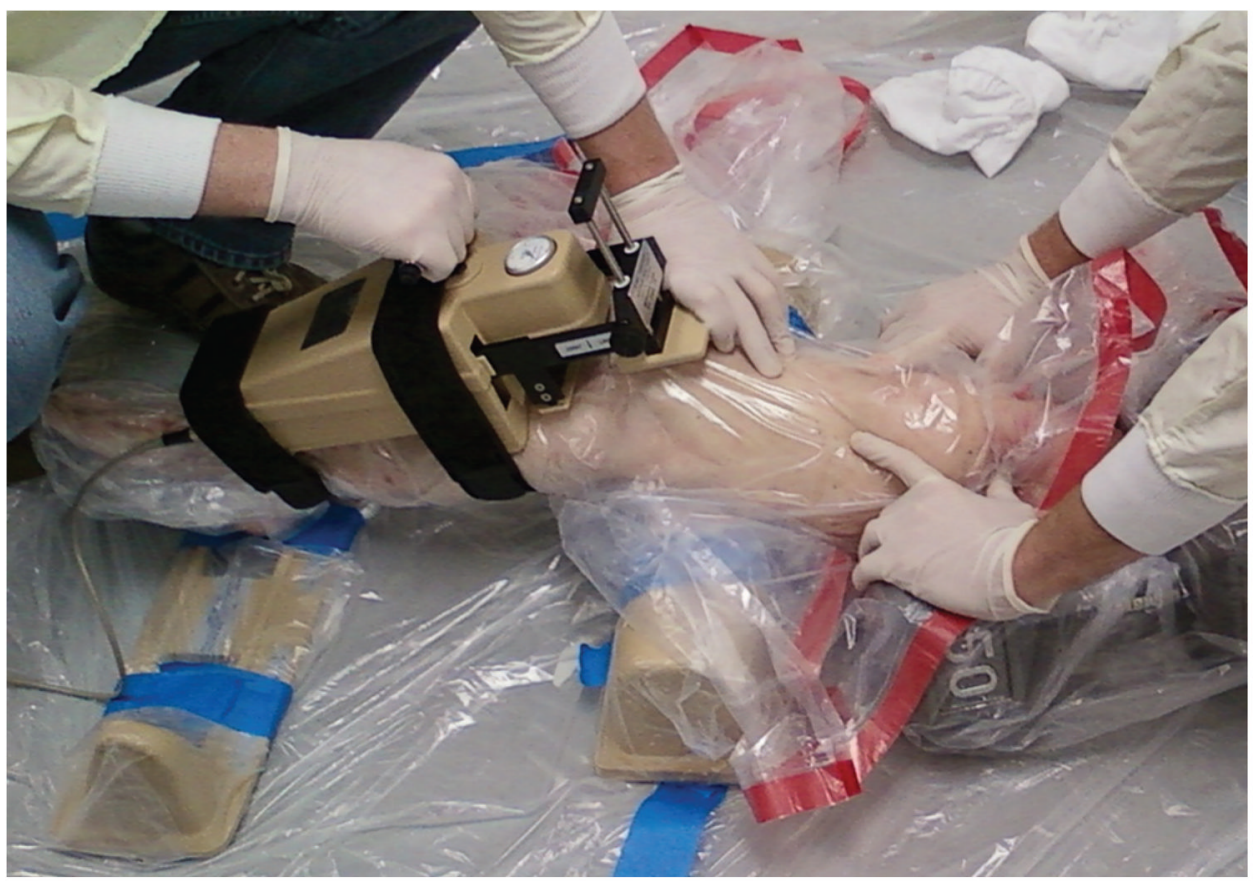

Figure 1.

The knee arthrometry procedure. 


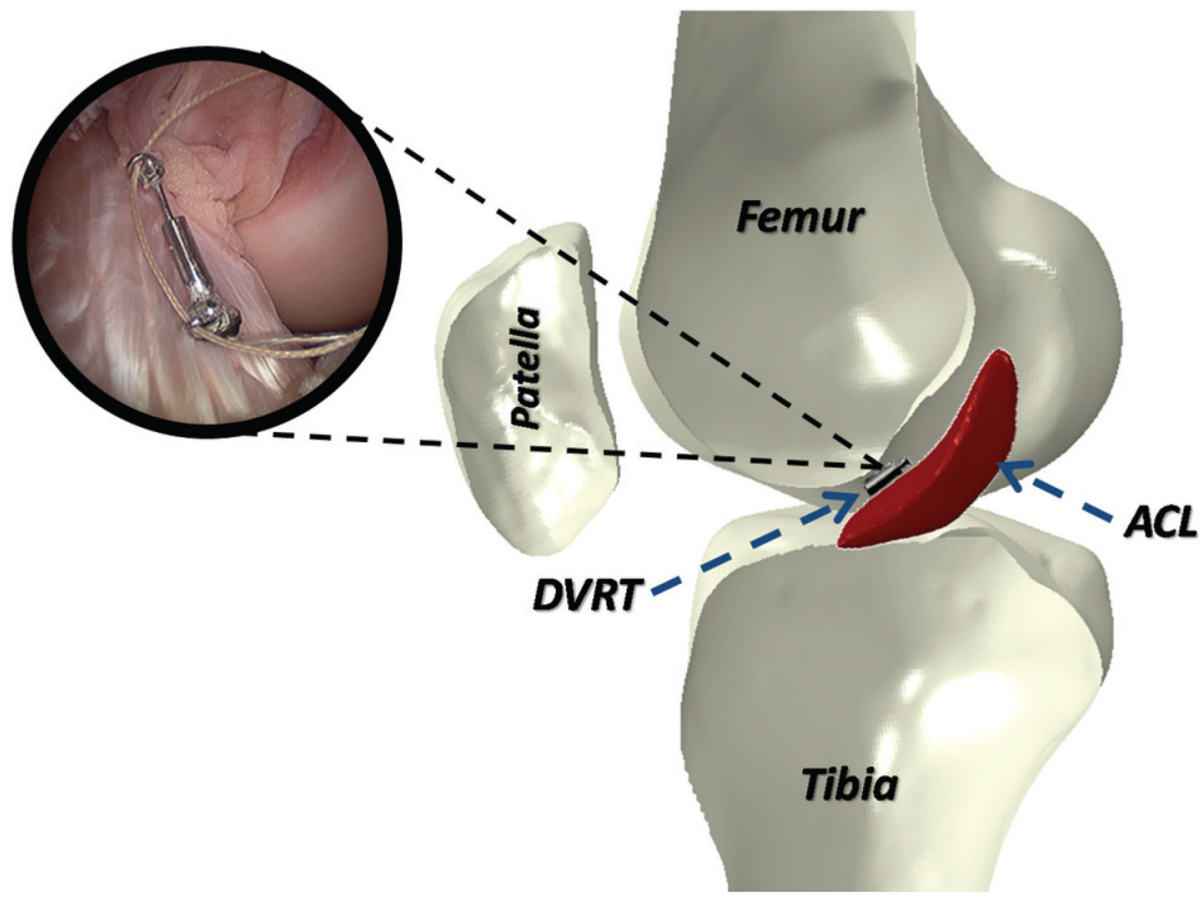

Figure 2.

Insertion of the differential variable reluctance transducer on the anteromedial bundle of the anterior cruciate ligament. 


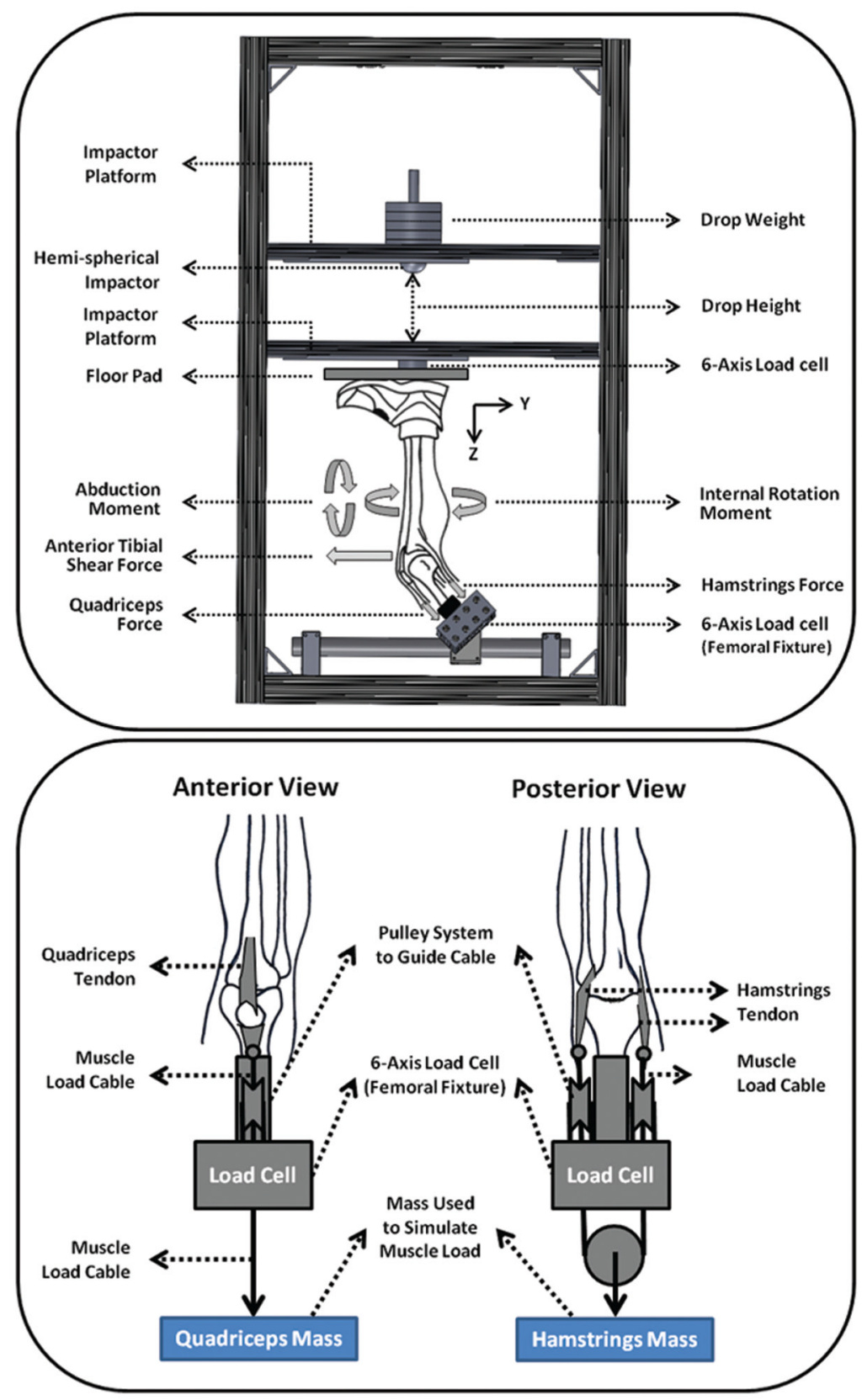

Figure 3.

Custom-designed drop-stand testing apparatus (top) and cable-pulley system used for the simulation of knee muscle forces (bottom). 

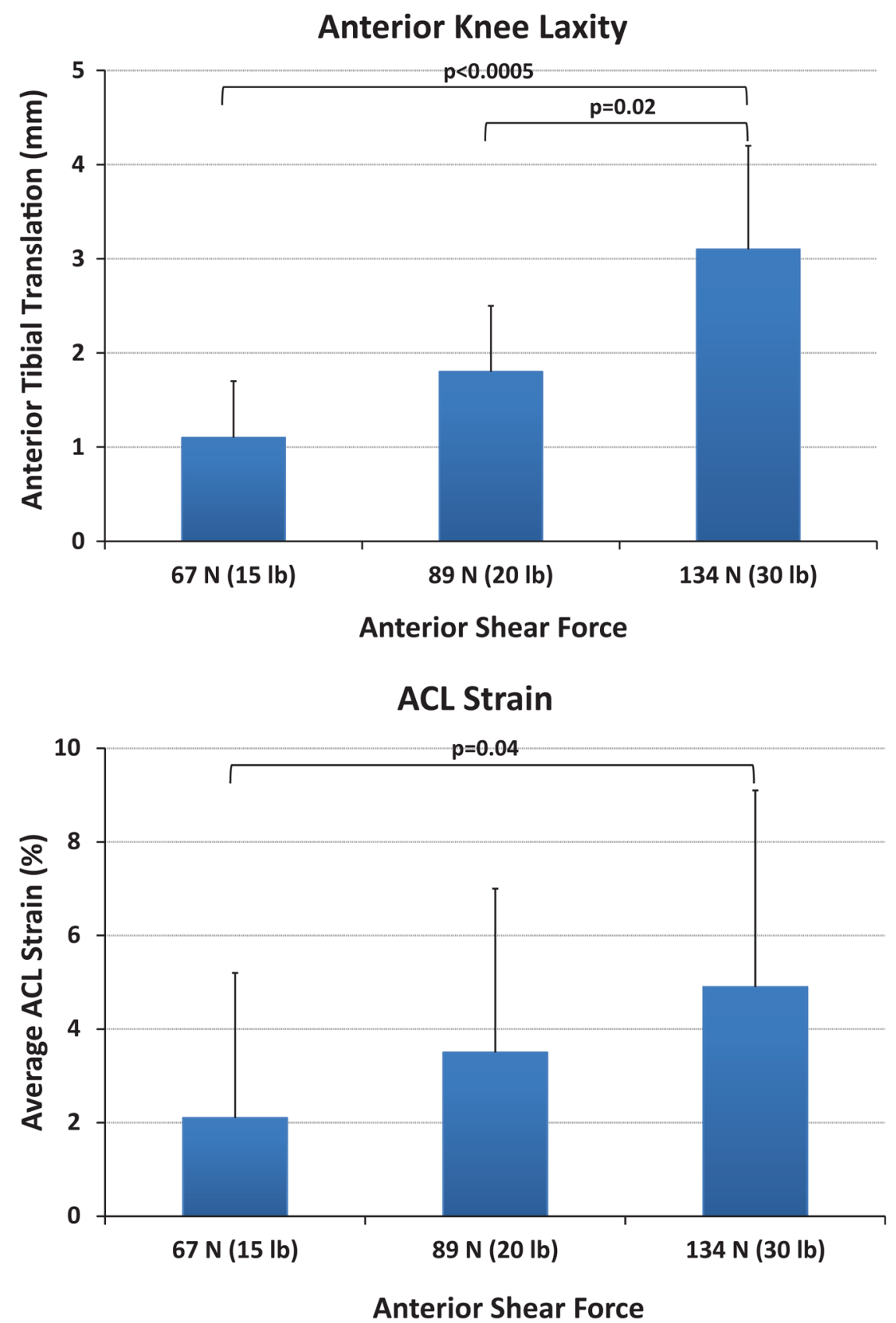

Figure 4.

Change in anterior knee laxity (top) and mean anterior cruciate ligament strain (bottom) under applied anterior shear force during the passive knee drawer test. 


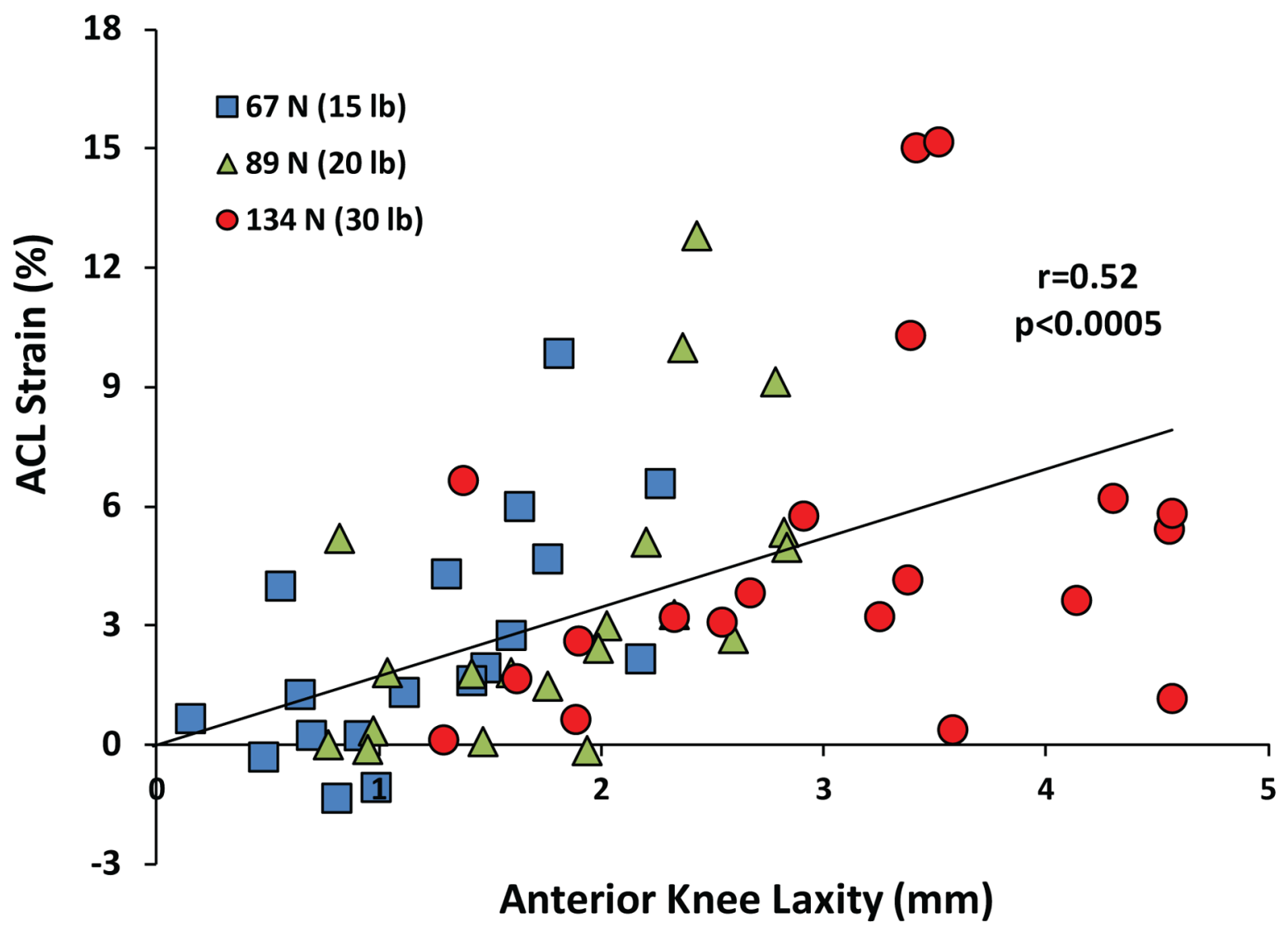

Figure 5.

Quantified anterior cruciate ligament strain versus anterior tibial translation during knee arthrometry. 
A

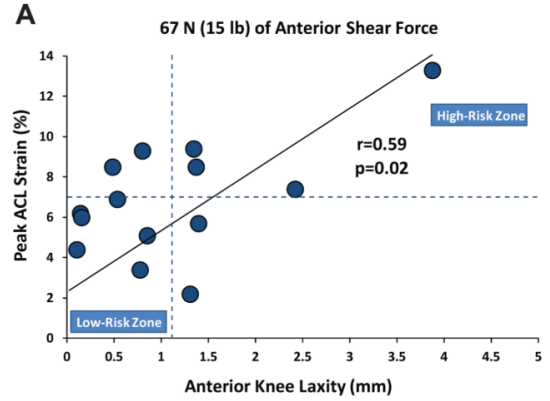

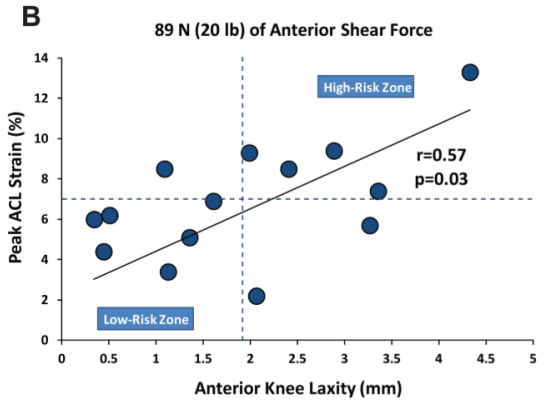

C

$34 \mathrm{~N}$ (30 lb) of Anterior Shear Force

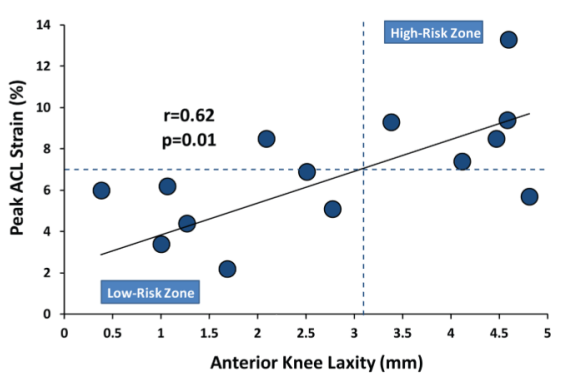

Figure 6.

Peak anterior cruciate ligament strain (during simulated bipedal landing) versus peak anterior tibial translation (quantified by the knee arthrometer) over a range of anterior shear loads. 
TABLE 1

Summary of Knee Arthrometry Data $(\mathrm{N}=20)^{a}$

\begin{tabular}{|c|c|c|c|}
\hline & \multicolumn{3}{|c|}{ Applied Anterior Shear Load } \\
\hline & $67 \mathrm{~N}(15 \mathrm{lb})$ & $89 \mathrm{~N}(20 \mathrm{lb})$ & $134 \mathrm{~N}(30 \mathrm{lb})$ \\
\hline ATT before DVRT insertion, $\mathrm{mm}$ & $1.1 \pm 1.0$ & $1.9 \pm 1.2$ & $2.8 \pm 1.4$ \\
\hline ATT after DVRT insertion, $\mathrm{mm}$ & $1.1 \pm 0.6$ & $1.8 \pm 0.7$ & $3.1 \pm 1.1$ \\
\hline ACL strain, \% & $2.1 \pm 3.1$ & $3.5 \pm 3.5$ & $4.9 \pm 4.3$ \\
\hline
\end{tabular}

${ }^{a}$ Values are expressed as mean \pm standard deviation. ACL, anterior cruciate ligament; ATT, anterior tibial translation; DVRT, differential variable reluctance transducer. 


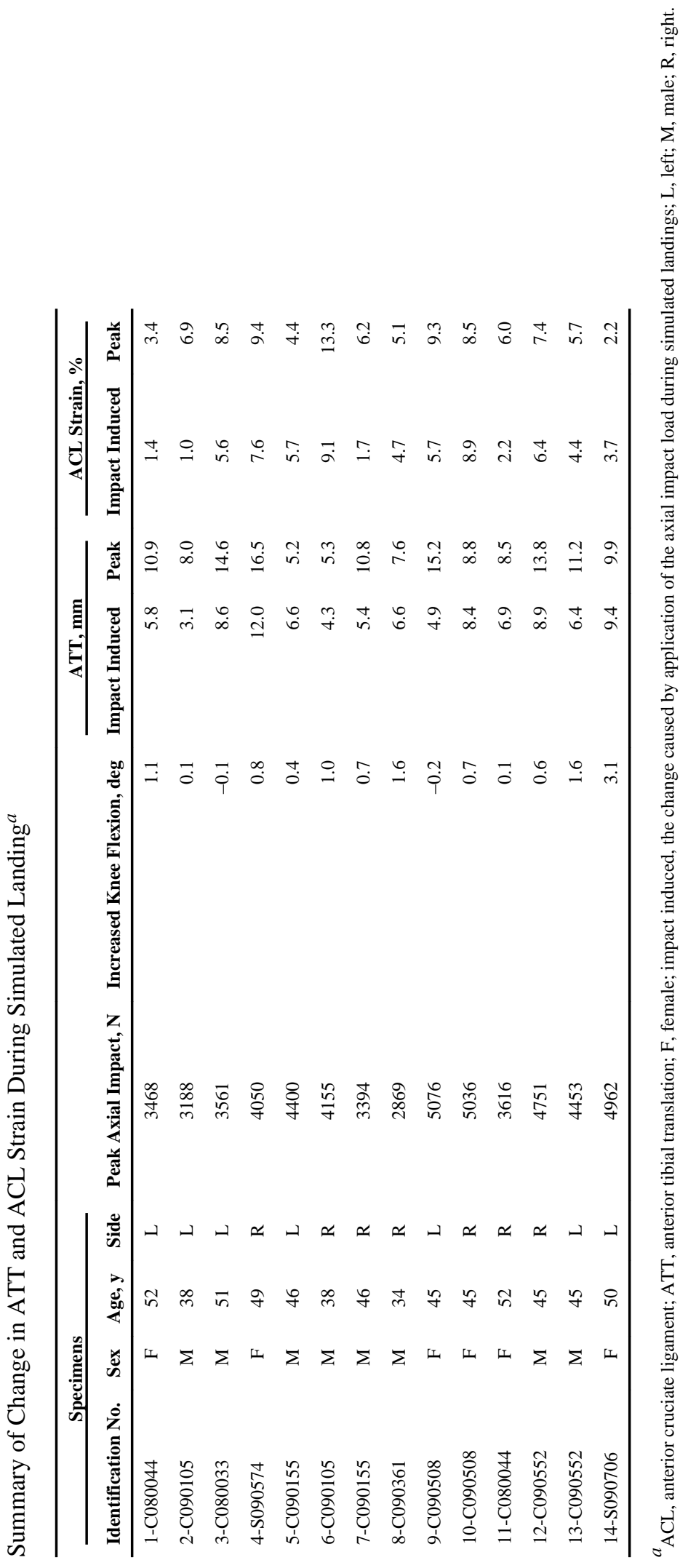

